\title{
Jnstrução Gramatical na Era da Tecnologia: investigando diferentes abordagens para o ensino-aprendizagem de Jnglês no Ensino Médio Jntegrado
}

\author{
Grammatical Instruction in THE TECHNOLOGICAL ERA: InVESTIGATING \\ DIFFERENT APPROACHES TO THE TEACHING AND LEARNING OF ENGLISH IN AN \\ INTEGRATED HIGH SCHOOL PROGRAM
}

Gicele Vergine PREBIANCA* Marli Fátima Vick VIEIRA** Kyria Rebeca FINARDI***

Resumo: O principal objetivo deste estudo é investigar o uso de recursos tecnológicos para o ensino-aprendizagem de Inglês como língua estrangeira (LE). Mais especificamente, o estudo pretende analisar o aprendizado de uma estrutura sintática por meio da instrução gramatical dada em dois ambientes: a sala de aula tradicional e o ambiente virtual de aprendizagem MOODLE. Outro objetivo do presente estudo é conhecer a percepção dos participantes e seu nível de satisfação, em relação às atividades para o ensino de gramática implementadas no MOODLE. Trinta alunos do Ensino Médio de um Instituto Federal, divididos em grupo controle e experimental, participaram do estudo. As análises estatísticas revelaram um bom desempenho

\footnotetext{
* Mestre e Doutora em Letras pela Universidade Federal de Santa Catarina. Instituto Federal de Educação, Ciência e Tecnologia Catarinense (IFC) - Campus Blumenau. Contato: gicele.prebianca@blumenau.ifc.edu.br.

** Mestre em Educação pela Universidade do Vale do Itajaí - UNIVALI (2008). Doutoranda em Educação. Professora efetiva do Instituto Federal de Educação, Ciência e Tecnologia Catarinense (IFC) - Campus Araquari. Contato: marlivickvieira@gmail.com. *** Mestre e Doutora em Letras pela Universidade Federal de Santa Catarina. Professora efetiva da Universidade Federal do Espírito Santo (UFES). Contato: kyria.finardi@gmail.com.
} 
dos participantes em ambos os testes de retenção da estrutura sintática alvo. O grupo controle superou o experimental em ambos os testes. Esses resultados são discutidos com base em teorias de processamento da informação e de aquisição de LE. A análise qualitativa sugere que, apesar de os participantes terem acesso a vários recursos tecnológicos, eles parecem não perceber a relevância de tais recursos para aprendizagem de uma LE. Em geral, os participantes apreciaram as atividades realizadas no MOODLE para o ensino de gramática.

Palavras-chave: Instrução gramatical. Ensino-aprendizagem de Inglês. Recursos tecnológicos.

\begin{abstract}
The main aim of the study is to investigate the contribution of the use of technological resources to teach English as a foreign language (FL). More specifically, the study focuses on the learning of a syntactic structure by means of grammatical instruction provided in two environments: a traditional classroom and a virtual learning environment - the MOODLE platform. A secondary aim is to assess participants' level of satisfaction concerning the activities implemented in MOODLE. Thirty students enrolled in the second year of the Technical High School Program at a Federal Institute, divided into control and experimental groups, participated in the study. Statistical analyses revealed that participants performed well in the tests for the retention of the syntactic structure. The control group outperformed the experimental one in both retention tests. Results are discussed in light of information processing and SLA theories. Regarding the qualitative analysis, data revealed that, although participants have access to several technological resources, they seem unable to perceive these resources as relevant to FL learning. The study also showed that, in general, participants enjoyed the activities carried out in the MOODLE platform.
\end{abstract}

Key words: Grammatical instruction. English teaching-learning. Technological resources.

\title{
Introdução
}

O rápido e crescente aumento de recursos tecnológicos disponíveis na sociedade atual, bem como a ampliação do acesso à informação e ao uso do computador por alunos em variados segmentos da sociedade, entre eles o da educação, tem motivado professores a repensar suas práticas pedagógicas. A 
revolução cibernética do último século também coloca em cheque a sala de aula tradicional de línguas, em particular aquelas em que a abordagem de ensino se limita à instrução face a face de itens gramaticais isolados de situações comunicativas, nas quais o foco é a troca de informação e a negociação de significados.

Os alunos que atualmente ocupam os bancos escolares, pertencentes, em sua grande maioria, à Geração $Z^{1}$, não concebem mais o mundo fora dos limites da grande rede, a internet, assim como precisam estar constantemente em contato com a tecnologia e seus artefatos. São esses alunos, com múltiplos saberes e experiências que nos desafiam a refletir sobre nossos papéis como educadores, e mais especificamente, sobre estratégias de ensino que ainda refletem o ensino conteudista tradicional do século passado. Como nos lembra Oliveira ([s.d.]), esses alunos “[...] usam computador antes mesmo da alfabetização", o que lhes permite o desenvolvimento de habilidades de caráter multifuncional. Porém, continuamos insistindo em ensinar-lhes como se nada soubessem.

Tendo em mente a necessidade de mudança na Educação em geral e no ensino de línguas, em particular, com a incorporação de novas tecnologias educacionais, o presente estudo investigou a contribuição do uso de recursos tecnológicos no ensino-aprendizagem de Inglês como língua estrangeira (doravante LE) no Ensino Médio Integrado². Para tanto, a investigação aqui reportada preocupou-se em analisar se o aprendizado (entendido, neste trabalho, como a retenção e o uso acurado da estrutura alvo na modalidade escrita) da estrutura sintática I wish + subject + past tense verb é influenciado pelo emprego de atividades realizadas em um ambiente híbrido ${ }^{3}$, que combina a

1 A Geração Z compreende, segundo Lauer (2011), “crianças e adolescentes que já nasceram em contato direto com a internet, a velocidade da informação e as novas tecnologias [...] A letra que dá nome a esse grupo vem do termo 'zapear', ato de trocar de canal de TV constantemente pelo controle remoto. Então, essa noção de troca rápida e constante batizou esse grupo etário."

2 O Ensino Médio Integrado refere-se, neste caso, à educação técnica profissional ministrada em conjunto com as disciplinas que compõem o núcleo básico de formação geral do Ensino Médio.

${ }^{3}$ Com base em Hinkelman e Gruba (2012) e Hong e Samimy (2010), conceituamos interação híbrida como a combinação de atividades mediadas pelo computador com a típica interação face a face de sala de aula. Um contexto de ensino-aprendizagem intrinsecamente multimodal, no qual as TICs e, em especial a internet, desempenham papéis fundamentais. 
abordagem tradicional de instrução face a face ${ }^{4}$ com atividades mediadas pelo computador, no ambiente virtual MOODLE 5 .

Outro objetivo do presente estudo, no tocante ao uso de recursos tecnológicos para aprender Inglês, é conhecer a percepção de alunos do Ensino Médio Integrado e seu nível de satisfação em relação às atividades realizadas no ambiente virtual de aprendizagem (plataforma) MOODLE para aprendizado de uma estrutura sintática.

$\mathrm{Na}$ próxima seção apresentamos os aportes teóricos que embasam esta pesquisa.

\section{Aportes Teóricos}

As primeiras abordagens para o ensino-aprendizagem de LE costumavam enfatizar a instrução gramatical direta, na qual regras de uso do idioma eram ensinadas isoladamente, fora de contextos significativos, como parte de uma grade de conteúdos, cujo foco, geralmente, não era o desenvolvimento das habilidades comunicativas dos aprendizes (WILLIS; WILLIS, 2001).

Mais recentemente, com o advento do método de ensino comunicativo (Communicative Language Teaching - CLT em inglês), passou-se a dar mais importância a práticas pedagógicas que priorizam o contexto de uso do idioma (MESKILL, 2005), resultando em atividades de sala de aula que se caracterizam pela interação entre os alunos, oportunidades para troca de informação, mais atenção ao conteúdo e significado das trocas interacionais e menos atenção ao aprendizado de regras e memorização de diálogos ou frases padronizadas (BRUMFIT, 1984; HOWATT, 1984, ambos citados em SPADA; LIGHTBOWN, 2008).

No entanto, o método comunicativo trouxe à tona outra questão: o papel da instrução gramatical no ensino comunicativo de uma LE (SPADA; LIGHTBOWN, 2008). Krashen (1985), por exemplo, defende que os alunos

${ }^{4}$ Neste artigo, interação face a face refere-se ao uso de metodologias de ensino tradicionais, em que predominam aulas expositivas em sala de aula.

5 MOODLE é o acrônimo de Modular Object-Oriented Dynamic Learning Environment. Também conhecido como ambiente virtual de aprendizagem, trata-se de um software livre, projetado para auxiliar educadores a criar cursos on-line com foco na interação e na construção coletiva do conteúdo (WIKIPEDIA, [s.d.]). 
podem aprender a LE independentemente do que acontece em sala de aula, de tal modo que a instrução gramatical não teria nenhum efeito sobre o aprendizado do idioma. Outros autores parecem acreditar que a instrução gramatical pode ter algum impacto na maneira como a LE é internalizada, dependendo do contexto de aprendizagem, da forma linguística a ser ensinada (simples, complexa, infrequente, para citar alguns exemplos), das características individuais de cada aprendiz e do estágio de desenvolvimento na aquisição da língua (ELLIS, 1991; LONG, 1991; DEKEYSER, 2000; DOUGHTY, 2003).

Long (1991) sugere dois tipos de instrução gramatical. O primeiro, por ele denominado Focus on Forms (FonFs), ou, em português, foco nas formas, tem o foco no ensino sistemático da estrutura linguística e geralmente guiado por um currículo organizado em torno de determinadas formas gramaticais isoladas, que devem ser ensinadas em uma ordem pré-determinada. Diferentemente do Focus on Forms, o outro tipo de instrução, definida por Long (1991) como Focus on Form (FonF), pressupõe o ensino de formas linguísticas que emergem das dificuldades que os alunos encontram ao se comunicar em uma LE. Ou seja, o ensino de determinada estrutura gramatical não é planejado no FonF, mas sim incidental.

Para Ellis (1994), há duas maneiras de implementar a abordagem FonF: por meio de atividades comunicativas que atentem à forma ou por meio de feedback corretivo que chame a atenção dos alunos para a forma linguística utilizada incorretamente no ato comunicativo. Independente da forma usada, parece haver o entendimento de que o FonF é compatível com o método comunicativo.

Em seu capítulo de livro intitulado 'Instrução formal e aquisição de segunda língua' (Formal instruction and second language acquisition), Ellis (1994) também discute outros dois tipos de instrução gramatical: a implícita e a explícita. Segundo o autor, a instrução implícita requer que o aluno induza as regras de uso da LE de exemplos e situações 'reais' de comunicação. Já a instrução explícita preconiza o ensino direto das regras gramaticais e a repetição da estrutura linguística ensinada para memorização da regra. Os resultados das pesquisas comparando a eficácia de ambos os tipos de instrução são inconclusivos, segundo Ellis (1994). Em geral, esses resultados mostram que a eficácia desses dois tipos de instrução parece depender do aspecto linguístico a ser ensinado e das características individuais de cada aprendiz, uma vez que, conforme sugerem as pesquisas, adultos parecem reter melhor a regra quando 
ela é apresentada explicitamente. Há, todavia, evidências de que alguns itens gramaticais são mais bem aprendidos por meio de uma abordagem implícita (ELLIS, 1994).

Finardi (2010), por exemplo, investigou a aquisição de uma estrutura sintática complexa em Inglês (L2) por falantes de Português Brasileiro (L1). A estrutura sintática investigada por Finardi (so + aux $+I$ e neither + aux $+I)$ não tem equivalente simples no Português e requer, para sua aquisição, a remarcação de parâmetros linguísticos (parameter resetting, em inglês) na língua mãe (L1). Finardi (2010) concluiu que, no caso da aquisição de estruturas sintáticas complexas, é necessário dar instrução gramatical focada na forma, de maneira explícita, a fim de garantir o aprendizado.

De acordo com Spada e Lightbown (2008), tanto as pesquisas envolvendo instrução gramatical quanto as experiências de professores em sala de aula de LE têm demonstrado que certos aspectos da língua só são aprendidos por meio de algum tipo de foco na forma (estrutura), uma vez que podem passar despercebidas durante a prática comunicativa do idioma. Assim, a instrução gramatical pode efetivamente melhorar o conhecimento da LE e o desempenho dos alunos no uso da língua quando incorpora atenção à forma estrutural da língua ao foco no significado, concomitantemente. $\mathrm{O}$ debate atual, de acordo com Spada e Lightbown (2008), não se concentra mais em determinar se a instrução gramatical em LE é ou não necessária no contexto comunicativo, mas como e quando ela é mais efetiva.

Spada e Lightbown (2008) usam os termos instrução com foco na forma isolada (isolated form-focused instruction, doravante IsFFI) e instrução com foco na forma integrada (integrated form-focused instruction, doravante InFFI) para se referir a diferentes maneiras de chamar a atenção dos alunos para o aprendizado de estruturas sintáticas da LE. A principal diferença entre esses dois tipos de instrução reside no fato de que a IsFFI implica em aprendizado intencional e explícito de algum item linguístico que, no entendimento do professor, é improvável que seja aprendido por meio de atividades essencialmente comunicativas. Apesar de a IsFFI compreender instrução explícita e atividades que não são necessariamente de natureza comunicativa, Spada e Lightbown (2008) defendem que esse tipo de instrução não é incompatível com um currículo baseado no método comunicativo. Já a InFFI ocorre em atividades de sala de aula cujo foco principal é a comunicação e a troca de significados entre os alunos. Diferentemente do Focus on Form proposto por Long (1991), Spada e Lightbown (2008) alegam que a InFFI contempla 
não só a instrução incidental, como também a planejada. Ou seja, pode ser intencionalmente preparada pelo professor como parte de uma atividade comunicativa.

A discussão sobre o melhor método para se ensinar uma LE ocupou linguistas aplicados por mais de uma década no final do século passado, sem, contudo, haver produzido um consenso. Várias também foram as variáveis discutidas como possíveis fatores impactantes no processo de aprendizado de um LE, tais como idade, sexo, aptidão e motivação, para citar algumas. Neste século, porém, parece que a preocupação maior dos linguistas aplicados não é qual o melhor método ou o impacto das variáveis citadas no aprendizado da língua, mas sim quais são as novas variáveis que podem afetar (e como podem afetar) o aprendizado de uma LE em um mundo altamente tecnológico, em que a forma como aprendemos é constantemente remodelada. Leffa (2005), por exemplo, questiona até se os instrumentos que usamos podem ser separados do que somos. A fim de refletir sobre a relação sujeito-instrumento ou ambiente, Leffa (2005) cita exemplos como o de um sujeito que usa lentes de contato ou um marca-passo, ou, ainda, um pino metálico no corpo. Podemos dizer que somos ou agimos da mesma forma sem a mediação (ou incorporação) desses artefatos tecnológicos e culturais que estão sendo radicalmente remodelados no nosso século? O mesmo podemos questionar a respeito dos novos recursos tecnológicos na educação do século XXI.

Nesse contexto e levando em conta o mundo internetizado (FINARDI; PREBIANCA; MOMM, 2013) em que vivemos, ousamos acrescentar, a esse debate, outra questão relevante que anda de mãos dadas com os novos artefatos tecnológicos que nos rodeiam: a relevância de uma abordagem híbrida que incorpore esses artefatos tecnológicos na instrução gramatical em LE. Nesse sentido, este estudo tentou responder à seguinte pergunta: a instrução gramatical, seja ela isolada (IsFFI) ou integrada (InFFI) (SPADA; LIGHTBOWN, 2008), com foco apenas na forma (Focus on Forms) ou na forma e no significado (Focus on Form) (LONG, 1991), implícita ou explícita (ELLIS, 1994), é afetada pelo tipo de abordagem (ambiente) utilizada, seja ela face a face ou híbrida?

Segundo Baturay, Daloglu e Yildirim (2010), apesar da incorporação massiva de recursos tecnológicos no ensino-aprendizagem de língua estrangeira na atualidade, pouca atenção tem sido dada ao ensino-aprendizagem de gramática mediado por computador. Chapelle $(1996,2007)$ nos lembra que, com o advento das novas tecnologias, são várias as opções de tarefas que os 
professores podem propor aos alunos a fim de impulsionar o aprendizado da língua. Como vimos, no caso da aquisição de estruturas sintáticas complexas, o foco na forma por meio de instrução explícita parece ser compatível com a prática de exercícios focados na forma mediados por computador, seja a instrução feita apenas por meio do uso do computador (CALL) ou ainda por meio de abordagens híbridas que combinam atividades online à instrução face a face.

Finardi e Reder (2013) conduziram um estudo com o objetivo de refletir sobre formas de desenvolver a competência comunicativa na LE por meio de abordagens híbridas de ensino, incorporando TICs como formas de aumentar o contato com a língua e cultura alvo, desenvolvendo, ao mesmo tempo, a autonomia do aprendiz de LE. Para tanto, o estudo descreveu um projeto de ensino que utilizou uma metodologia híbrida aplicada a estudantes do nono ano de uma escola pública em Vila Velha, no Espírito Santo. A metodologia do projeto incorporou o uso de vídeos, atividades no laboratório de informática da escola - acessando um curso no laboratório virtual da Universidade do Oregon (ANVILL) -, interação em uma rede social e o uso de um software. Os resultados do projeto de ensino sugerem que abordagens híbridas que incorporam TICs promovem maior motivação e autonomia no aluno, além de propiciarem o desenvolvimento da competência comunicativa na língua estrangeira e do letramento digital.

Em sua extensa revisão sobre aprendizado híbrido, Cardoso (2012) defende a necessidade de mais pesquisas que analisem a implementação da abordagem híbrida em ambientes formais de aprendizagem de LE. De acordo com a autora, é importante conhecer qual ou quais combinações multimodais oferecem maior potencial para o ensino-aprendizagem de Inglês. Nesse sentido, o presente estudo representa uma tentativa de documentar, metodológica e experimentalmente, como a abordagem híbrida pode moldar o aprendizado de gramática em LE, oferecendo uma contribuição para a linguística aplicada em geral e, mais especificamente, para a formação e educação continuada de professores de LE para o século XXI.

\section{Metodologia}

O estudo é de cunho quase experimental, uma vez que os participantes foram selecionados levando-se em consideração o ano do Ensino Médio em que estão matriculados. A análise dos dados é de natureza mista (DORNYEI, 2007), uma vez que utiliza dados quantitativos e qualitativos. 
Trinta e dois alunos do $2^{\circ}$ ano do Ensino Médio Técnico Integrado em Agropecuária e do Ensino Médio Técnico Integrado em Informática de um Instituto Federal foram divididos em dois grupos: controle e experimental. Enquanto o grupo controle foi composto apenas por alunos do curso técnico em Informática (17), o grupo experimental combinou alunos do curso técnico em Informática (6) e alunos do curso técnico em Agropecuária (9). Todos os alunos, por sua vez, foram submetidos a um pré-teste (Anexo 1) para garantir que não conheciam a estrutura alvo a ser ensinada. Apenas os alunos cuja pontuação no pré-teste foi zero foram selecionados para prosseguir na pesquisa, reduzindo a amostra a trinta participantes. Assim, os dois grupos foram compostos por 15 participantes.

O grupo controle recebeu instrução formal sobre o uso da estrutura sintática I wish + subject + past tense verb por meio de uma abordagem tradicional de ensino (instrução explícita e isolada - face-to-face), que compreendeu aula expositiva e exercícios gramaticais escritos e individuais em sala de aula. $\mathrm{O}$ grupo experimental, além de receber o mesmo tratamento da abordagem tradicional dada ao grupo controle, participou de outras atividades pedagógicas envolvendo instrução gramatical e prática (Anexo 2), utilizando a plataforma MOODLE no laboratório de informática da Instituição, durante 3 aulas de 45 minutos cada da disciplina de Inglês. Essa abordagem foi chamada de abordagem híbrida.

A escolha da estrutura alvo foi motivada por uma observação, de cunho pedagógico, de que o aprendizado dessa estrutura sintática costuma ser problemático para falantes de Português como L1, uma vez que sua utilização correta na produção escrita em Inglês (L2) parece ser garantida apenas por meio do foco explícito na forma. Assim, o presente estudo contribui para uma melhor compreensão do impacto da instrução explícita em L2, em geral, e na aquisição dessa estrutura sintática alvo em particular.

Logo após receberem a instrução, um teste com o objetivo de medir a retenção da estrutura sintática alvo na produção escrita dos alunos participantes foi aplicado - o teste de retenção imediata (TRI) (Anexo 1). Aproximadamente trinta dias após o primeiro teste, um segundo teste escrito foi administrado com o intuito de verificar se a retenção da estrutura sintática alvo ainda persistia - o teste de retenção consecutiva (TRC) (Anexo 1).

Os resultados dos testes de RI e de RC foram submetidos a procedimentos estatísticos, a fim de investigar se havia uma diferença significativa entre o grupo controle e o grupo experimental em relação à 
retenção da estrutura sintática alvo. No presente estudo, a retenção foi operacionalizada como o uso acurado da estrutura alvo na modalidade escrita, representado pelo número total de acertos de cada teste.

Ao final do estudo, os 15 participantes do grupo experimental responderam a um questionário com 5 perguntas abertas, que visou a coletar suas percepções e nível de satisfação a respeito da abordagem híbrida para o ensino de gramática e das atividades mediadas pelo computador, implementadas na plataforma MOODLE (o Anexo 2 lista as atividades utilizadas no ambiente virtual).

\section{Resultados e Discussão}

\subsection{Análise quantitativa}

A fim de avaliar se houve uma diferença estatisticamente significativa entre o teste de retenção imediata (TRI) e o teste de retenção consecutiva (TRC), o teste Wilcoxon Signed Rank (W) foi aplicado aos dados. A Tabela 1 apresenta os resultados desse teste.

Tabela 1 - Resultado do teste comparativo entre retenção imediata e retenção consecutiva

\begin{tabular}{lcc}
\hline Testes de retenção & $\mathrm{N}$ & Mean Rank \\
\hline Imediata (TRI) & 30 & 16.30 \\
Consecutiva (TRC) & 30 & 6.88 \\
\hline Test Statistics & TRC-TRI & \\
\hline $\mathrm{Z}$ & -4.112 & \\
Asymp. Sig. & $.000^{*}$ & \\
\hline${ }_{\mathrm{P}<0,05}$ & &
\end{tabular}

A análise dos dados revelou que as medianas em ambas as condições de testagem diferiram significativamente, $\mathrm{z}=-4.11, \mathrm{p}=.000$. A mediana dos ranques em favor da retenção imediata (RI) foi 16.30 enquanto o da retenção consecutiva (RC) foi 6.88 . Em outras palavras, os participantes se saíram 
melhor no teste de retenção imediata do que no teste de retenção consecutiva, talvez por terem a fórmula da estrutura alvo (conhecimento declarativo) mais ativa na memória. Nessa mesma linha, outra explicação plausível para esse resultado é a falta de prática (necessária para desenvolver o conhecimento procedimental) na estrutura alvo durante um tempo que pode ter sido relativamente longo para esquecer a regra (conhecimento declarativo), já que o teste consecutivo foi aplicado aproximadamente trinta dias após o teste de retenção imediata. Além disso, é provável que a instrução focada na forma tenha fornecido conhecimento declarativo suficiente para realizar o teste imediato de forma mais acurada, porém pouco conhecimento procedimental para realizar o teste consecutivo.

Abordagens baseadas na Teoria do Processamento da Informação no ensino-aprendizagem de LE entendem a prática como sendo um elemento-chave na transformação de conhecimento declarativo em procedimental (McLAUGHLIN, 1987; DEKEYSER, 1998, 2007). Se isso não ocorreu no intervalo entre testagens, parece plausível propor a hipótese de que, em geral, os participantes tiveram mais dificuldade em aplicar procedimentos mais automatizados, necessários para usar corretamente a estrutura sintática alvo no teste de retenção consecutiva. Nessa visão, com o passar do tempo, parece ser mais difícil automatizar conhecimento em uma LE sem a prática ou ensino apropriados, o que pode ter ocorrido durante o período que antecedeu o teste de retenção consecutiva. Também é importante notar que a estrutura alvo investigada não tem um equivalente perfeito no Português (L1), o que pode ter causado erros durante a performance, como será discutido mais adiante.

O teste Wilcoxon também confirmou a hipótese de que qualquer tipo de retenção da estrutura alvo (imediata ou consecutiva) seria resultado da instrução gramatical, fosse ela de forma isolada na abordagem face a face ou na abordagem híbrida. Como esperado, as medianas dos testes de RI e de $\mathrm{RC}$ foram significativamente diferentes das medianas dos pré-testes, $\mathrm{z}=$ $-4.789, \mathrm{p}=.000$ e $\mathrm{z}=-4.716, \mathrm{p}=.000$, respectivamente, como pode ser observado na Tabela 2. 
Tabela 2 - Resultado do teste comparativo entre pré-teste, retenção imediata e retenção consecutiva

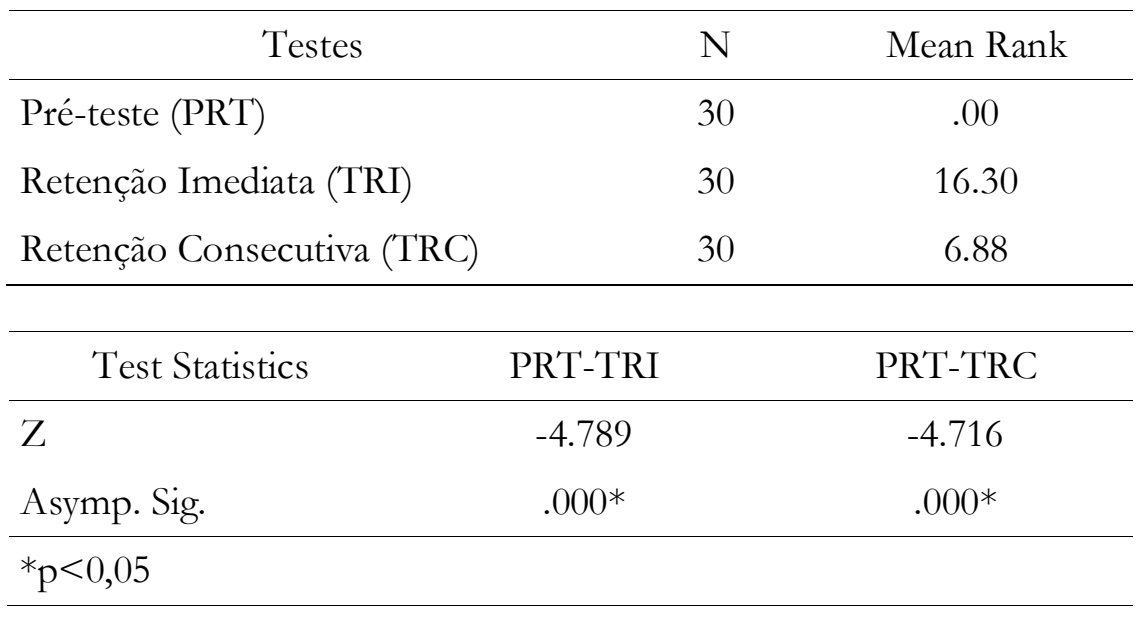

Este resultado corrobora Finardi (2010), que mostrou que a retenção da estrutura sintática $S_{0}+a u x+I$ e Neither $+a u x+I$ foi causada pelo tratamento (instrução focada na forma durante aulas presenciais) dado aos alunos de Inglês participantes do estudo. Finardi (2010) também sugere que, no caso de estruturas sintáticas complexas (que não têm equivalente perfeito entre L1 e L2, por exemplo), que exigem a remarcação de parâmetros na $\mathrm{L}^{6}{ }^{6}$, a instrução explícita e focada na forma é necessária para sua aquisição. Este resultado também vai ao encontro da alegação de Spada e Lightbown (2008), de que a instrução focada na forma isolada pode ser usada por professores em situações de ensino nas quais certas características da língua-alvo exijam instrução explícita para sua aquisição, tal como no caso da estrutura sintática investigada neste estudo.

O uso da estrutura sintática I wish + subject + past tense verb parece qualificar como uma estrutura que requer instrução explícita na forma, já que no

${ }^{6}$ De forma simples, a remarcação de parâmetros em L1 envolve notar que um dado parâmetro em L2 não corresponde ao parâmetro empregado em L1, requerendo a reorganização da gramática de L2 em formação, a fim de incorporar esse conhecimento. 
Português Brasileiro (L1) não se usam equivalentes perfeitos nem a tradução literal da estrutura sintática alvo em construções como: 'Eu desejo que eu tivesse um carro' (I wish I had a car) ou 'Eu desejo que minha casa fosse maior' (I wish my house were bigger), mesclando tempos presente e passado para se referir ao significado factual, o de que a pessoa não tem um carro ou que sua casa é muito pequena, por exemplo. Em outras palavras, o tempo verbal no passado na cláusula subordinada ( had no caso do primeiro exemplo e were no segundo) não se relaciona com o significado de tempo nesses tipos de construções sintáticas, mas, antes, à factualidade, já que I had a car and my house were bigger expressam significados contra factuais sob a influência de wish (RODNEY; PULLUM, 2002).

No sistema inflexional do Português Brasileiro, o verbo wish parece manter sua referência de tempo (por exemplo, 'eu desejo ter um carro' ou 'eu desejo ter uma casa maior’) e, portanto, essa questão de falta de correspondência entre o tempo e o verbo não parece ser um problema para falantes do Português Brasileiro se comunicando em L1. Entretanto, em L2, isso pode levá-los a construir frases com cláusulas subordinadas no presente, transferindo, assim, padrões sintáticos da L1 para a performance em L2, como, em I wish I have a car ou I wish my house is bigger. Outro aspecto importante, neste caso, é que as frases como I wish I had a car e Eu desejo ter um carro, mesmo se sintaticamente equivalentes, parecem não carregar o mesmo tom semântico. Enquanto a primeira induz a pensar em um evento que é improvável de acontecer no presente ou futuro próximo, a segunda nos dá a impressão de que ter um carro é algo realmente possível naquele momento.

Devido a essas incongruências entre os padrões sintáticos das L1 e L2 em relação ao uso da estrutura I wish + subject + past tense verb, acreditamos que qualquer retenção da estrutura alvo só possa ser possível por meio da instrução explícita e focada na forma isolada; fato este corroborado pelos resultados do presente estudo reportados até o momento.

Ainda que o procedimento Wilcoxon tenha mostrado que a performance no TRI tenha sido melhor do que no TRC, não é possível afirmar qual grupo (controle ou experimental) teve a melhor performance. Assim, o teste Mann-Whitney U foi usado para avaliar a hipótese de que o grupo experimental teria uma melhor performance, em média, do que o grupo controle em ambas as condições de testagem (TRI e TRC), como consequência de ter experimentado a abordagem híbrida.Os resultados desse teste são reportados na Tabela 3 . 
Tabela 3 - Comparação entre os grupos controle e experimental no TRI e no TRC

Diferença entre grupos

(Mean Rank)

\begin{tabular}{lccc}
\hline & $\mathrm{U}$ & $\mathrm{z}$ & $\begin{array}{c}\text { Sig. } \\
\text { (2-tailed) }\end{array}$ \\
\cline { 2 - 4 } $\begin{array}{l}\text { TRI } \\
\text { Controle X Experimental } \\
\quad \text { TRC }\end{array}$ & 90.0 & -.939 & .348 \\
Controle X Experimental & & & \\
& 41.0 & -2.997 & $.003^{*}$ \\
$\begin{array}{l}\mathrm{N}=15 \text { (em cada grupo: } \\
\text { controle e experimental) } \\
* \mathrm{p}<0,05\end{array}$ & & & \\
\hline
\end{tabular}

Os resultados do teste Mann-Whitney rejeitaram essa hipótese, já que não houve uma diferença estatisticamente significativa entre os grupos experimental e controle no TRI, $\mathrm{z}=-.939, \mathrm{p}>$.005. Porém, a média de ranque do grupo controle foi estatisticamente superior ao do grupo experimental no TRC, $\mathrm{z}=-2.997, \mathrm{p}=.003$.

Considerando o fato de que os participantes não tinham conhecimento procedimental suficiente para usar a estrutura alvo corretamente, esperava-se que ambos os grupos (controle e experimental) tivessem uma performance similar em ambas condições de testagem imediata e consecutiva, o que, claramente, não foi observado neste estudo. Apesar de os participantes serem todos alunos do Ensino Médio na mesma instituição, especula-se que o grupo controle pode ter sido composto de aprendizes mais proficientes em Inglês e mais digitalmente letrados do que os participantes do grupo experimental, em razão do curso que ambos faziam, uma vez que o grupo controle era composto apenas de alunos do curso técnico em Informática enquanto o grupo experimental era composto de uma maioria de alunos do curso em Agropecuária (9). Assim, quaisquer efeitos que o grupo experimental pudesse ter tido no ambiente híbrido podem ter sido compensados ou mascarados 
pela melhor proficiência no Inglês e familiaridade com o ambiente virtual do grupo controle. Ou seja, o curso técnico em Informática pode ter dado, aos participantes do grupo controle, uma vantagem sobre os participantes do grupo experimental, composto por alunos de ambos os cursos.

Outra possível explicação para esse resultado pode se relacionar com as atividades realizadas na plataforma MOODLE. As atividades implementadas no MOODLE foram selecionadas levando em consideração os diferentes estilos de aprendizagem que, geralmente, se fazem presentes em uma sala de aula. Ou seja, tentou-se utilizar atividades que, de certa forma, fossem interessantes para o maior número de alunos possível e pudessem ser compatíveis com as diferentes formas de aprender. No entanto, tendo em vista os resultados supracitados, parece plausível levantar a hipótese de que algumas dessas atividades não tenham sido apropriadas para fomentar o aprendizado de uma estrutura sintática complexa, que requer remarcação de parâmetros linguísticos em L1 (por exemplo FINARDI, 2010), como visto anteriormente nesse artigo.

As atividades D e E (ver Anexo 2), por exemplo, parecem estar mais ligadas a uma instrução gramatical implícita, na qual o aluno deve induzir a regra por meio de exemplos (ELLIS, 1994) e cujos efeitos no aprendizado de uma estrutura sintática provavelmente não seriam evidentes a curto prazo. A estrutura gramatical analisada neste estudo parece requerer um tipo de instrução gramatical explícita, por meio da qual o aluno possa aplicar a fórmula da forma declarativa (em outras palavras, a regra $I$ wish + subject + past tense verb), visualizando a diferença de uso dessa estrutura na L1 e na L2, reestabelecendo, assim, os parâmetros necessários para utilizar a regra adequadamente na LE. Finardi (2010) revisa modelos linguísticos (por exemplo WHITE, 1985, 1991, 1992) de aquisição de LE (L2), que entendem que a L1 influencia o processamento e a aquisição de uma L2. Evidências da complexidade do processamento de certas estruturas sintáticas em L2, dependendo da remarcação de parâmetros linguísticos na L1, também podem ser encontrados no livro de Gass e Schachter (1989). Com base nesses modelos, Flynn $(1987,1989)$ sugere que aprendizes de L2 usam princípios de organização sintática da L1 para a construção da gramática da L2. Quando esses princípios envolvem a remarcação de parâmetros linguísticos, os aprendizes de L2 devem reconhecer a congruência ou incongruência desses parâmetros entre a L1 e a L2 por meio da instrução focada na forma. Quando esses parâmetros diferem entre si, a aquisição de L2 pode ser interrompida ou dificultada, uma vez que 
os aprendizes têm que determinar novos parâmetros e valores para construir a gramática em L2. Quando os parâmetros são semelhantes, o aprendizado é facilitado. Assim, a instrução explícita focada na forma seria necessária, principalmente para aprender estruturas sintáticas cujos valores em L1 diferem dos valores em L2.

Uma última explicação para o resultado obtido com o teste MannWhitney pode estar relacionada ao fato de que a exposição dos participantes do grupo experimental às atividades no MOODLE limitou-se a apenas 3 aulas, de 45 minutos cada, da disciplina de Inglês. A limitação do número de aulas para a abordagem híbrida justifica-se pela dificuldade em efetivar reservas nos laboratórios de informática da instituição de ensino, quando se trata de uma disciplina que não compõe o núcleo profissionalizante do curso de Ensino Médio Técnico Integrado em Informática. Esse tempo de apenas 2 horas e 25 minutos de atividades na plataforma MOODLE pode não ter sido suficiente para impactar o aprendizado da regra da estrutura alvo (conhecimento declarativo) e o uso (conhecimento procedimental) da mesma, gerando diferenças significativas entre os dois grupos. Vale ressaltar que, nesse contexto, entendemos o conhecimento declarativo da estrutura sintática como sendo o conhecimento da regra I wish + subject + past tense verb, e o conhecimento procedimental como a correta aplicação da regra em frases na modalidade escrita.

\subsection{Análise qualitativa}

Com o intuito de investigar a percepção de alunos do Ensino Médio Integrado no que tange ao uso da tecnologia para aprender Inglês, o presente estudo analisou o nível de satisfação dos participantes em relação às atividades para o ensino de gramática, implementadas no ambiente virtual conhecido como plataforma MOODLE. A análise dos dados do questionário aplicado aos alunos do grupo experimental é apresentada a seguir.

Pergunta 1: Qual sua opinião sobre a abordagem de ensino que mesclou atividades em sala de aula e atividades na plataforma MOODLE para a aprendizagem da estrutura gramatical I wish + subject + past tente verb que estudamos neste semestre?

A maioria dos participantes achou a abordagem híbrida interessante, alegando que ajudou na aprendizagem da LE. Apenas um dos 15 participantes 
alegou não achar que essa abordagem tenha ajudado no aprendizado. Os participantes disseram, ainda, que as atividades foram interativas e que gostaram das atividades realizadas no MOODLE. Cinco participantes responderam que preferem a plataforma MOODLE ao uso do livro didático por ser diferente e mais motivadora.

Pergunta 2: Qual atividade da plataforma MOODLE você mais gostou de realizar e por quê?

A maioria dos participantes respondeu que a atividade que mais gostou foi a de completar, seguida da atividade dos super-heróis, da música e, finalmente, da atividade de escolher a alternativa correta. Dois alunos disseram que não gostaram de nenhuma atividade realizada no MOODLE, e um afirmou que prefere provas às atividades.

Pergunta 3: Antes de utilizar a plataforma MOODLE durante as aulas de Inglês, você já havia tido contato com a mesma? Em que situação?

Em relação aos alunos do curso técnico em Agropecuária, a maioria afirmou que desconhecia a plataforma. Poucos disseram que já haviam tido contato com MOODLE, utilizando-o para a resolução de listas de exercícios de matemática e para atividades de cursos de Inglês paralelos à escola. Em contrapartida, entre os alunos do curso técnico em Informática, apenas dois (2) disseram não ter tido contato com o MOODLE antes do estudo; os demais responderam que já haviam usado o ambiente virtual nas aulas do Ensino Técnico, especialmente como ferramenta para entrega de trabalhos.

Este resultado corrobora nossa especulação ao explicar a análise comparativa entre os grupos experimental e controle em ambos os testes de retenção da estrutura alvo, de que a diferença a favor do grupo controle pode ter sido mediada pela composição dos grupos, já que o grupo experimental tinha mais alunos do curso em Agropecuária (9) do que em Informática (6), e o grupo controle, ao contrário, era composto apenas de alunos do curso técnico em Informática (15). Conforme vimos na resposta à pergunta 3, os alunos do curso em Informática já tinham familiaridade com o MOODLE, ao contrário do que ocorreu com os alunos do curso em Agropecuária. Da mesma forma, podemos especular que os alunos do curso em Informática tinham mais familiaridade com o Inglês, já que, no curso, muitos dos termos técnicos de informática são nesse idioma. 
Pergunta 4: Costuma realizar atividades na internet para aprender Inglês? Quais?

Quando questionados se costumavam utilizar atividades na internet para aprender Inglês, a maioria dos alunos de ambos os cursos afirmou não usar a rede com essa finalidade. Dos 15 alunos do curso médio técnico em Agropecuária, apenas cinco alunos afirmaram que realizam atividades na internet para a prática do idioma. Outros 10 alunos não possuem esse hábito. Os alunos que afirmaram usar a internet para aprender Inglês disseram que a utilizam para baixar e traduzir música; acessar as redes sociais; acessar sites de outros países; acessar sites da escola de Inglês para realizar atividades do curso; ler textos em inglês; jogar em inglês e pesquisar no Google tradutor.

As respostas dos alunos do curso médio técnico em Informática a esta pergunta não foram muito diferentes. Apenas quatro alunos responderam que utilizam ferramentas disponíveis na internet para aprender a língua inglesa fora da sala de aula. Esses alunos afirmaram que costumam jogar em inglês; ouvir e traduzir músicas; acessar as redes sociais para se comunicar no idioma e acessar o site da escola de Inglês para resolver atividades on-line. Os demais alunos alegaram não usar a internet para aprender inglês. Vale lembrar, entretanto, que o fato de os alunos de Informática não usarem ferramentas na internet para aprender Inglês não significa, como vimos, que eles usem menos internet, significa apenas que não a usam com esse fim. Como podemos observar na resposta à pergunta 3 , os alunos do curso de Informática têm mais familiaridade com a internet e com o MOODLE do que os alunos do curso de Agropecuária.

Pergunta 5: Como você acha que aprende Inglês melhor? Cite algumas estratégias que você gosta de utilizar.

Entre os alunos do curso médio técnico em Agropecuária, as estratégias citadas para o aprendizado de Inglês foram, em ordem de preferência: tradução de músicas; filmes em inglês; redes sociais; jogos de vídeo game; exercícios na internet; vídeo aula e técnicas de conversação.

Já os alunos do curso médio técnico em Informática citaram, em ordem de preferência: ouvir músicas; assistir a filmes e seriados; comunicarse usando as redes sociais; usar chats para conversação; realizar exercícios na Internet; jogar vídeo game e ter aulas formais.

De certa forma, a análise das estratégias preferidas dos alunos vem ao encontro do perfil de uso da internet para aprender inglês, já reportado na 
resposta à pergunta 4. Em suma, os alunos parecem não perceber a internet como fonte de recursos para o aprendizado do idioma. Em outras palavras, eles não a reconhecem como ferramenta legítima de aprendizado do idioma, fato esse que parece claro se observarmos que, apesar de toda influência tecnológica do mundo contemporâneo nas mais variadas esferas sociais, entre elas a escola, os alunos continuam acreditando que ouvir e traduzir letras de música é a melhor estratégia para se aprender inglês.

Reiterando, este estudo teve como principal objetivo analisar o aprendizado de uma estrutura sintática em inglês por meio da instrução gramatical dada em dois ambientes: na sala de aula tradicional e no ambiente virtual de aprendizagem MOODLE. De forma geral, a análise quantitativa mostrou que a estrutura sintática investigada neste estudo pode ser vista como uma estrutura complexa, por exigir remarcação de parâmetros linguísticos e que, por sua vez, seu aprendizado requer uma instrução focada na forma de maneira explícita e sistemática. Os testes estatísticos, porém, não confirmaram a principal hipótese levantada no estudo, de que a instrução gramatical com foco na forma no ambiente híbrido seria mais eficiente do que a instrução tradicional com foco na forma em sala de aula. Os possíveis motivos sugeridos para justificar esse resultado referem-se ao perfil dos participantes que compunham os grupos controle e experimental, às características das atividades realizadas no MOODLE, bem como ao tempo de exposição à abordagem híbrida.

O objetivo secundário do presente estudo era conhecer a percepção dos participantes e seu nível de satisfação em relação às atividades para o ensino de gramática implementadas no ambiente virtual. A análise qualitativa revelou que, apesar de os alunos terem apreciado a instrução gramatical no ambiente híbrido, a grande maioria não utiliza recursos da internet para aprender inglês e tampouco a percebe como uma ferramenta legítima, potencializadora do processo.

\section{Conclusão}

A análise quantitativa do presente estudo revelou três resultados principais. Primeiro, a retenção da estrutura sintática alvo I wish + subject + past tense verb deu-se por conta do tratamento empregado, ou seja, da instrução gramatical aplicada a ambos os grupos. Segundo, os alunos apresentaram 
melhor desempenho no teste de retenção imediata. Terceiro, o grupo controle superou o grupo experimental no teste de retenção consecutiva. Esses resultados nos conduzem às seguintes conclusões:

$\Rightarrow$ Algum tipo de instrução gramatical (seja ela face a face ou híbrida) parece ser necessário para a retenção e o uso correto de estruturas sintáticas complexas em L2. O estudo sugere ainda que a instrução focada na forma de maneira explícita é a melhor opção no caso de estruturas sintáticas que requeiram a remarcação de parâmetros em L2;

$\Rightarrow$ Os escores do teste de retenção imediata parecem refletir um estágio mais declarativo no aprendizado da LE, e a prática parece ser um fatorchave para a procedimentalização da língua;

A análise qualitativa, por outro lado, revelou um importante resultado: apesar de toda influência tecnológica vivenciada no mundo atual e da incorporação das tecnologias a todos os espaços e atividades do dia a dia, os alunos ainda não concebem o uso dessas tecnologias como uma forma legítima de aprendizado de Inglês.

Sendo assim, conclui-se que:

$\Rightarrow \mathrm{O}$ uso de recursos tecnológicos, especialmente aqueles providos pela internet, deve ser constantemente questionado em face às reais necessidades de uso desses recursos e às ideologias e posições culturais implícitas no comportamento de nossos alunos;

$\Rightarrow$ Os recursos tecnológicos devem ser cuidadosamente selecionados e avaliados antes de serem incorporados à rotina pedagógica, tendo em vista o aspecto do idioma que se pretende trabalhar e o resultado esperado.

No tocante à análise qualitativa, os resultados sugerem que, apesar de os alunos terem acesso aos recursos tecnológicos providos pela internet, parecem não perceber a relevância de tais recursos para aprendizagem de LE. Um exemplo disso é o fato de que poucos participantes afirmaram usar a rede para a prática e aprendizagem do idioma. Em outras palavras, o amplo uso da internet para fins de entretenimento e interação social parece não garantir aos alunos a percepção do potencial da rede para o aprimoramento da língua-alvo.

Sugestões para futuras pesquisas compreendem: (i) investigar uma amostra maior de participantes; (ii) investigar a retenção da mesma estrutura sintática alvo em diferentes níveis de proficiência, a fim de verificar se os escores de retenção imediata e consecutiva permanecem estáveis ao longo do aprendizado da LE; e (iii) controlar o nível de proficiência na LE e 
familiaridade com TICs dos participantes, a fim de não mascarar possíveis resultados do uso de ferramentas tecnológicas e abordagens híbridas para o aprendizado do idioma.

Em suma, acreditamos que o uso das TICs no Ensino Médio Integrado, assim como em outras modalidades de ensino, não deve representar apenas um modismo ou mais uma estratégia motivacional. Sendo assim, não se trata apenas de utilizar as mais modernas tecnologias, mas de reconhecer quando e quais recursos incorporar à sala de aula presencial e por quê. A fim de ajudar professores a tomar decisões informadas sobre o uso de TICs, o presente estudo espera ter contribuído com a análise e reflexão aqui realizada.

Outro importante fator para o sucesso no uso de recursos tecnológicos está ligado à forma como os alunos encaram o uso da tecnologia na esfera educacional e suas possíveis contribuições para o processo de ensinoaprendizagem como um todo. Nesse sentido, é essential que o professor de LE leve em consideração a real necessidade da incorporação das TICs no ensino presencial, a fim de evitar "inovações conservadoras" (CYSNEIROS, 1999) e de promover a apropriação das novas formas de pensar da contemporaneidade.

\section{Anexo 1 - Testes}

\section{$\Rightarrow$ Pré-teste}

Complete as frases de acordo com suas expectativas, usando WISH:

1. My car is old

2. My house is small

3. My eyes are blak

4. I have only one brother

5. I don't have an apartment

6. I don't have a computer

7. My family is big

8. My computer is slow

9. My watch is brown

10. I don't have good books

11. I can't speak Spanish

12. I can't drive 
$\Rightarrow$ Teste de Retenção Imediata (TRI)

Complete as frases de acordo com suas expectativas, usando WISH:

1. I don't have a big house

2. I don't have a new car

3. I am short

4. I have two sisters.

5. I don't have a dog.

6. My apartment is old

7. My room is not clean

8. I can't swim

9. I don't dance very well

10. I can't play volleyball

$\Rightarrow \quad$ Teste de Retenção Consecutiva (TRC)

Complete as frases de acordo com suas expectativas, usando WISH:

1. I can't speak Italian

2. My cell phone is not modern

3. I don't have a computer

4. My classroom is small

5. My friends are not nice

6. My tennis shoes are not comfortable

7. My house is big

8. I don't have a good job

9. I don't have a car

10. I don't have beautiful glasses

Anexo 2 - Atividades desempenhadas na plataforma MOODLE

\section{A) Regra de uso da estrutura sintática: I Wish I ... Expressing Wishes}

I Wish I Had - Present Wishes

The phrase I wish I had ... is used to imagine a present situation. Here are some common phrases with I wish I had ... 


\section{Examples:}

I wish I had more money.

I wish I had more free time.

I wish I had more friends.

I wish I had a better car.

In the phrase, I wish I had ... 'had' is the past simple form of the verb 'to have'. You can certainly use other verbs with 'I wish ...'.

\section{Examples:}

I wish I spoke Russian.

I wish I played the guitar

I wish I drove a Mercedes.

I wish I lived in Seattle.

The use of the I wish I had ... is very similar to the second conditional because it expresses a situation which is contrary to fact. Look at these sentences comparing the two forms:

\section{Examples}

I wish I had a bigger house. = If I had more money, I would have a bigger house. I wish I knew more people in this town. = If I smiled more, I would meet more people.

I wish I had a driver's license. = If I were 16, I would have a driver's license.

\section{B) Completando as frases}

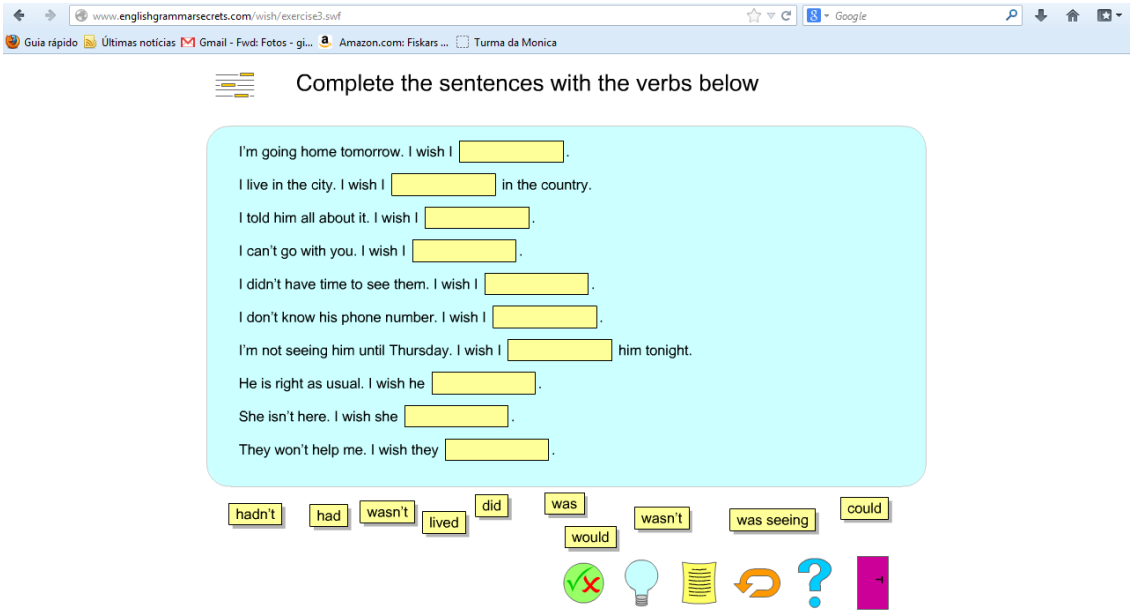




\section{C) Encontrando a resposta correta}

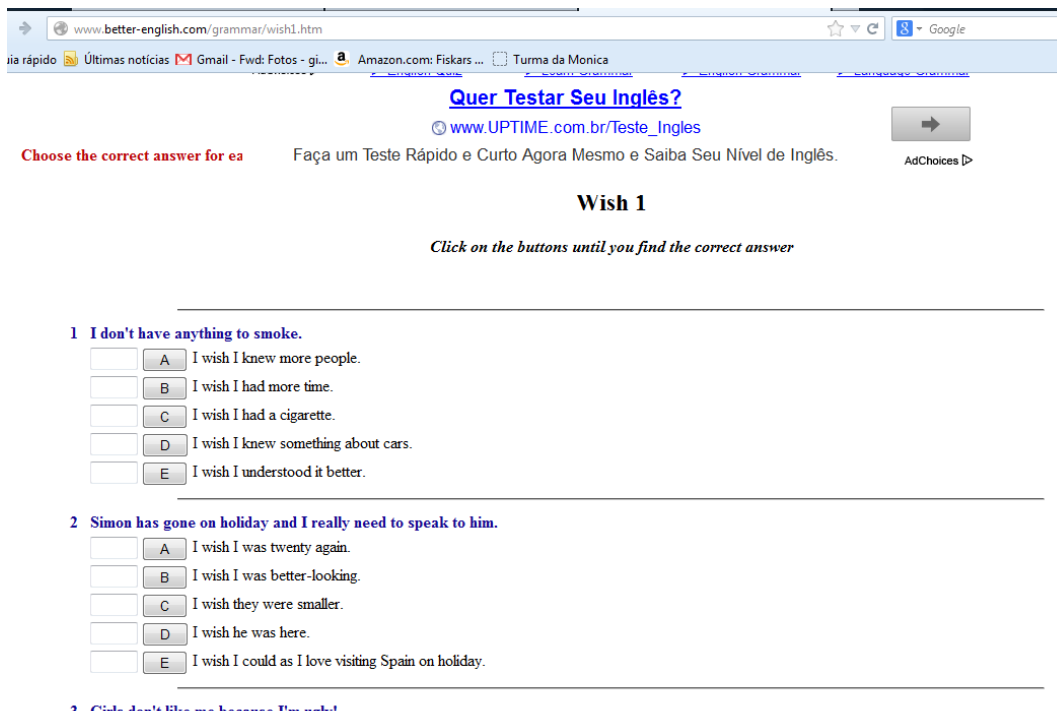

3 Girls don't like me because I'm ugly!

\section{D) Ouvindo a história}

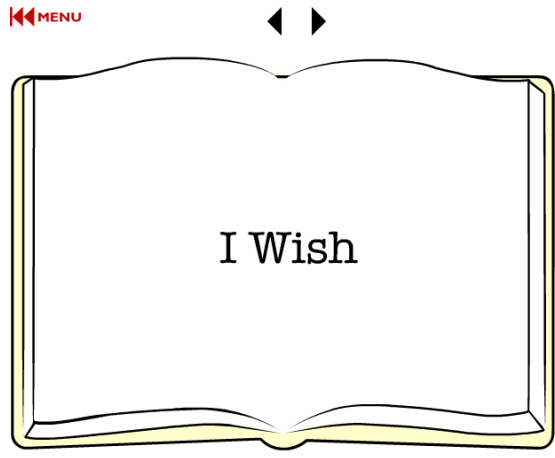

\section{Audio Script for the story:}

There was a carterpillar crawling on a leaf

One Day it saw a bird flying in the Sky. "I wish I could fly" - it said 
And it saw a dragonfly flying around. "I wish I could fly"- it said The carterpillar was sad and hungry. It ate and ate.

It grew big and fat. "I'm so sleepy"- it said

So, the carterpillar made a shell to sleep in. "I wish I could fly" - it dreamed.

Some days latter, the cartepillar woke up.

"Look at my wings!", said the carterpillar.

It became a beautifl butterfly.

\section{E) Completando as lacunas da música}

Song: I wish I had an angel (by Nightwish)

http://www.youtube.com/watch?v=KCSj_qUsKlo

I wish I had an angel

For one of love

I wish I had your angel tonight

Deep into a day

I took a step outside an heart

Prepare to hate me fall when I may

This will hurt you like never before

Old loves they die hard

Old they die harder

I wish I had an angel

For one of love

I wish I had your angel

Your Virgin Mary undone

I'm in with my lust

Burning angel wings to

I wish I had your angel tonight

I'm going so frail and cruel

Drunken disguise changes all the

Old loves they die hard Old they die harder

I wish I had an angel for one of love

I wish I had your angel

Your Virgin Mary undone 
I'm in with my lust

Burning angel wings to

I wish I had your angel tonight

Greatest thrill

Not to kill

But to have the prize of the night

Hypocrite

Wannabe friend

13th disciple who betrayed me for nothing!

Last dance, first

Your touch, my bliss

I wish I had an angel always comes with dark thoughts

For one of love

I wish I had your angel

Your Virgin Mary undone

I'm in with my lust

Burning angel wings to

I wish I had your angel tonight

I wish I had an angel

I wish I had an angel

I wish I had an angel

I wish I had an Angel

F) Expressando desejos sobre super poderes: I wish I ...

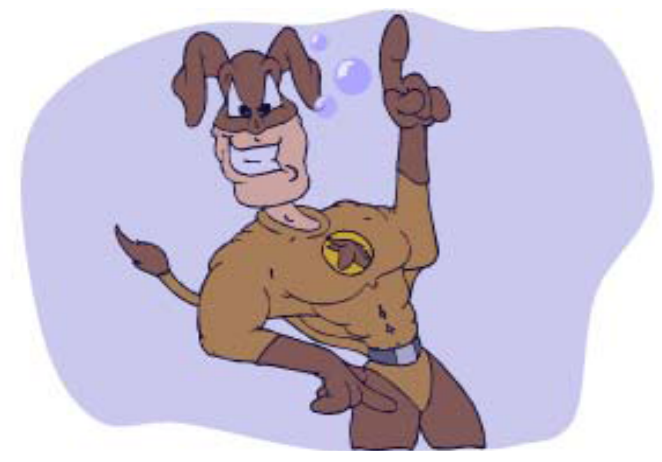

breathe under water 


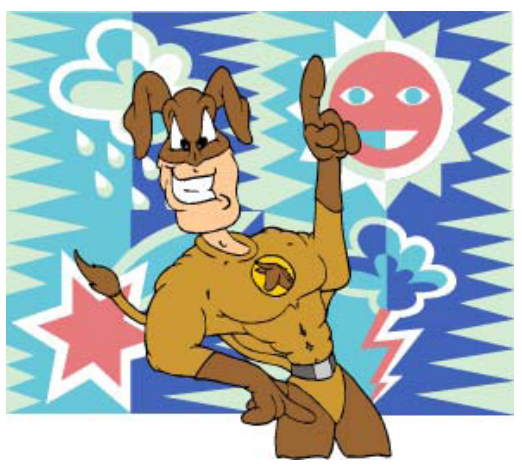

control the weather

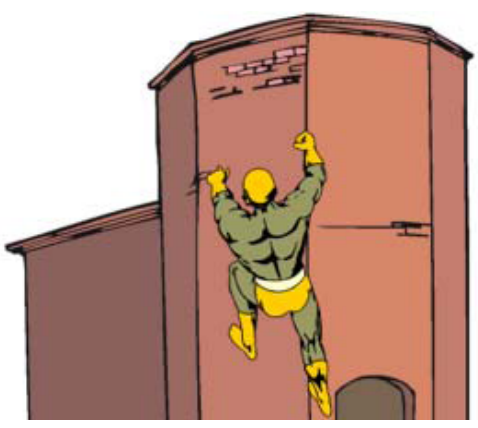

climb walls

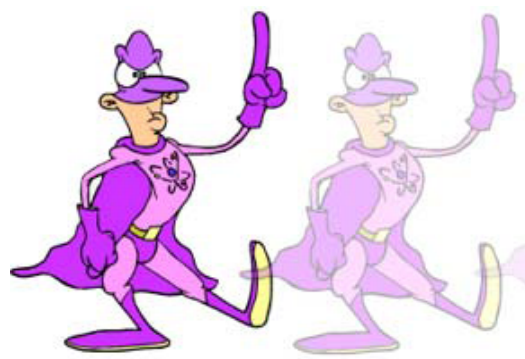

become invisible 


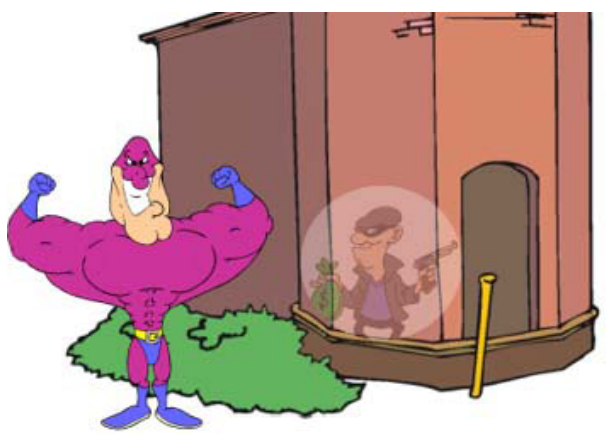

see through walls

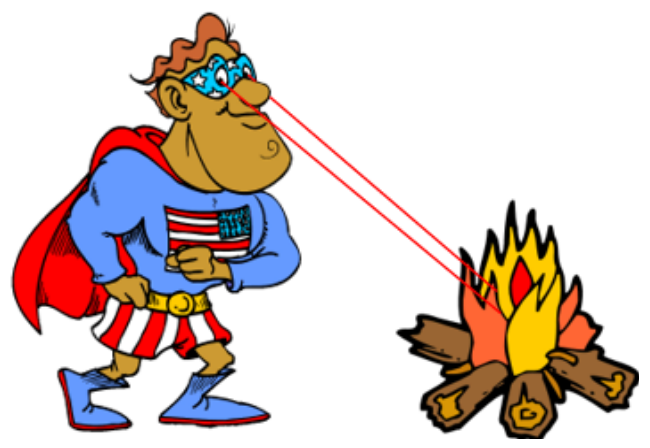

shoot lasers
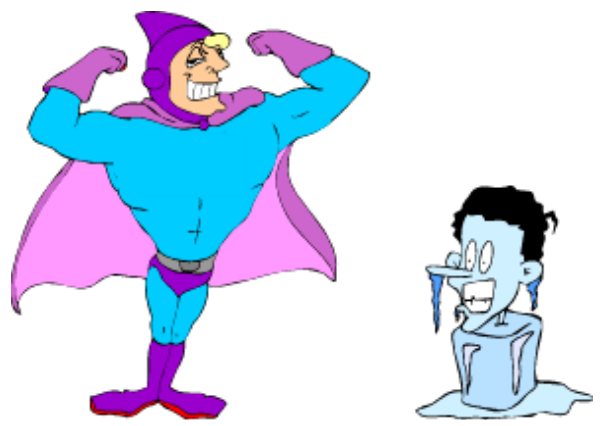

freeze things 


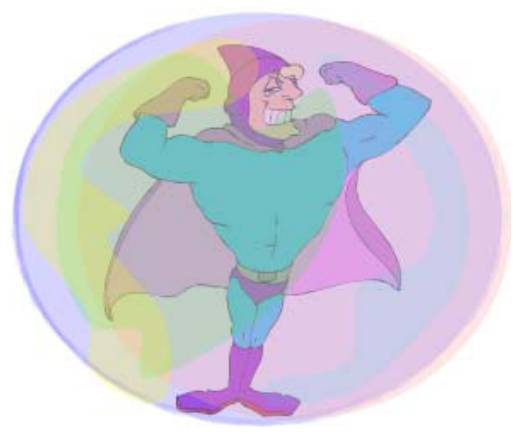

make force fields

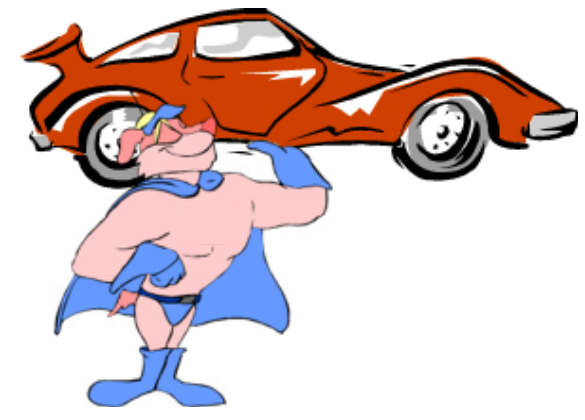

lift anything

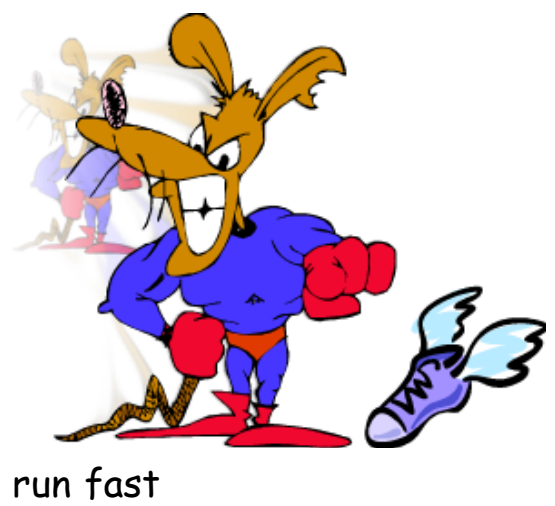




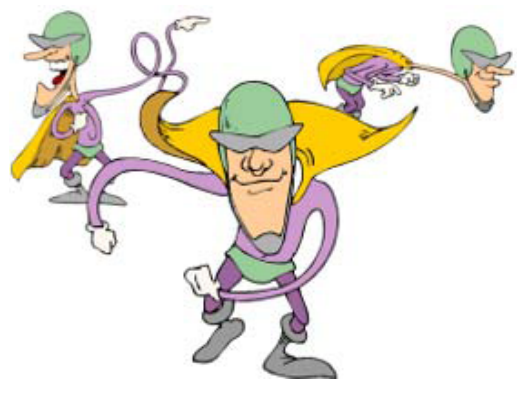

stretch very far

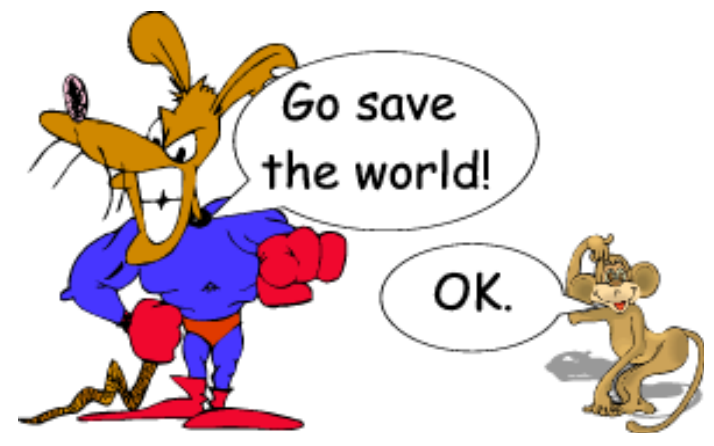

talk to animals

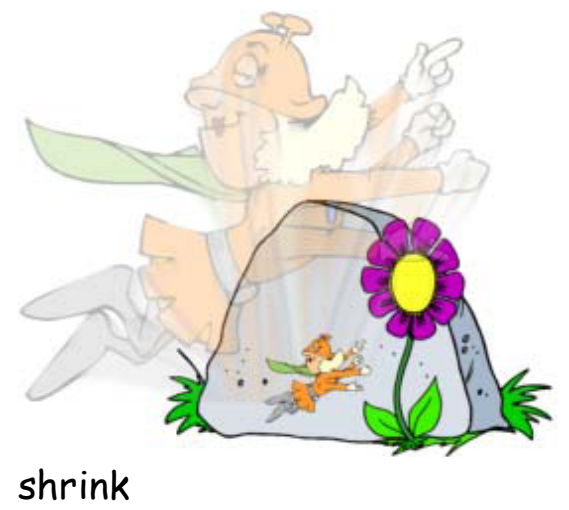




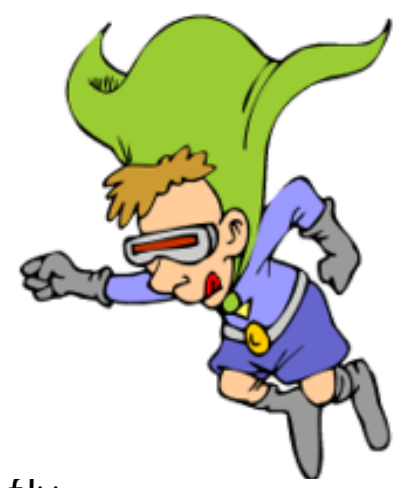

tly

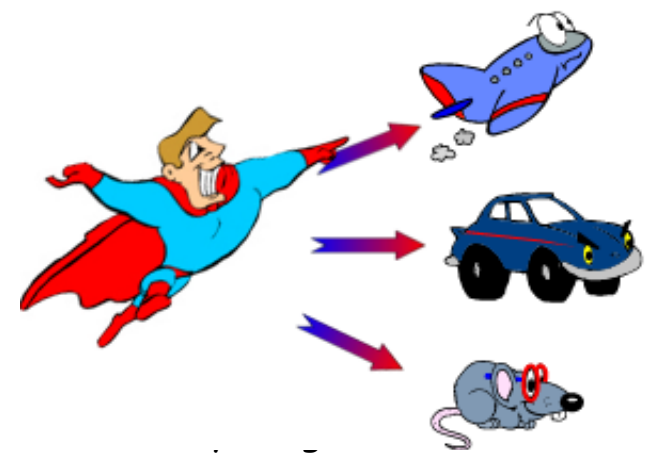

\section{Referências}

BATURAY, M.; DALOGLU, A.; YILDIRIM, S. Language practice with multimedia supported web-based grammar revision material. ReCALL, v. 22, n. 3, p. 313-331, 2010.

CARDOSO, G. L. The effects of CALL on L2 vocabulary acquisition: An exploratory study. 2012. Tese (Doutorado em Letras/Inglês) Universidade Federal de Santa Catarina, Florianópolis.

CHAPELLE, C. CALL-English as a second language. Annual Review of Applied Linguistics, v. 16, p.139-157, 1996. 
CHAPELLE, C. Technology and second language acquisition. Annual Review of Applied Linguistics, v. 27, p. 98-114, 2007.

CYSNEIROS, P. G. Novas tecnologias na sala de aula: melhoria do ensino ou inovação conservadora? Informática Educativa, v. 12, n. 1, p.11-24, 1999.

DEKEYSER, R. Beyond focus on form: Cognitive perspectives on learning and practicing second language grammar. In: WILLIAMS, C. D. J. (Ed.). Focus on form in classroom second language acquisition. Cambridge, UK:

Cambridge University Press, 1998. p. 42-63.

DEKEYSER, R. M. The robustness of critical period effects in second language acquisition. Studies in Second Language Acquisition, v. 22, p. 493-533, 2000.

DEKEYSER, R. Practice in a second language: Perspectives from applied linguistics and cognitive psychology. Cambridge: Cambridge, 2007.

DOUGHTY, C. Instructed SLA: Constraints, compensation, and enhancement. 202 TESOL QUARTERLY. In: DOUGHTY, C. J.; LONG, M. H. (Eds.). The handbook of second language acquisition. Malden, MA: Blackwell, 2003. p. 256-310.

DORNYEI, Z. Research methods in Applied Linguistics. New York, USA: Oxford University Press, 2007.

ELLIS, R. Second language acquisition and language pedagogy. Clevedon: Multilingual Matters, 1991.

ELLIS, R. The study of second language acquisition. Oxford, UK: Oxford University Press, 1994.

FINARDI, K. Working memory capacity in second language learning. Saarbrucken: Lambert Academic Publishing, 2010.

FINARDI, K.; PREBIANCA, G. V. V.; MOMM, C. F. Tecnologia na Educação: o caso da internet e do inglês como linguagens de inclusão. Cadernos do IL, n. 46, p. 193-208, 2013.

FINARDI, K.; REDER, J. TICs e interação transcultural no ensino de L2. Contextos Linguisticos, v. 7, p. 254-272, 2013. 
FLYNN, S. A parameter-setting model of L2 acquisition. Dordrecht: Reidel,1987.

FLYNN, S. The role of the head-initial/head-final parameter in the acquisition of English relative clauses by adult Spanish and Japanese speakers. In: GASS, S.; SCHACHTER, J. (Eds.). Linguistic perspectives on second language acquisition. Cambridge: Cambridge Applied Linguistics, 1989. p. 89108.

GASS, S., SCHACHTER, J. Linguistic perspectives on second language acquisition. Cambridge: Cambridge University Press. 1989.

HINKELMAN, D.; GRUBA, P. Power within blended language learning programs in Japan. Language Learning \& Technology, v. 16, n. 2, p. 46-64, 2012.

HONG, K. H.; SAMIMY, K. K. The influence of L2 teachers' use of CALL modes on language learners' reactions to blended learning. CALICO Journal, v. 27, n. 2, p. 328-348, 2010.

KRASHEN, S. D. The input hypothesis: Issues and implications. New York: Longman, 1985.

LEFFA, V. J. On becoming digitally literate: The production of computermediated materials by language teachers. In: CONVENÇÃO DA ASSOCIAÇÃO DOS PROFESSORES DE INGLÊS DO RIO GRANDE DO SUL, 2005, Porto Alegre. Teaching and Learning Processes. Porto Alegre: PUC-RS, 2005. (CD-Rom).

LONG, M. Focus on form: A design feature in language teaching methodology. In: DE BOT, K.; GINSBERG, R.; KRAMSCH, C. (Eds.). Foreign language research in cross-cultural perspective. Amsterdam: John Benjamins, 1991. p. 39-52.

McLAUGHKIN, B. Theories of second-language learning. London: Edward Arnold Publishers, 1987.

MESKILL, C. Triadic scaffolds: Tools for teaching English language learners with computers. Language, Learning, \& Technology, v. 9, n. 1, p. 46-59, 2005. 
OLIVEIRA, S. Geração Z se conecta antes de saber ler. [s.d.]. Disponível em: <http://www.sidneioliveira.com.br/samba/Imprensa/folha-de-saopaulo.html>. Acesso em: 3 jul. 2013.

RODNEY, H.; PULLUM, G. K. A short overview of English syntax.

Cambridge, UK: Cambridge University Press, 2002.

SPADA, N.; LIGHTBOWN, P. M. Form-focused instruction: isolated or integrated? TESOL Quarterly v. 42, p. 181-207, 2008.

WHITE, L. The 'Pro-drop' parameter in adult second language acquisition. Language Learning, v. 35, n. 1, p. 47-62, 1985.

WHITE, L. Adverb placement in second language acquisition: Some effects of positive and negative evidence in the classroom. Second Language Research, v. 7, p. 133-161, 1991.

WHITE, L. Long and short verb movement in second language acquisition. Canadian Journal of Linguistics, v. 37, n. 2, p. 273-286, 1992.

WILLIS, D.; WILLIS, J. Task-based language learning. In: CARTER, R.; NUNAN, D. (Orgs.). Teaching English to speakers of other languages.

Cambridge: CUP, 2001. p. 173-179.

Recebido em: 03/02/2014 Aceito em: 02/06/2014 